\title{
Desplazamiento de los Puntos de Referencia Craneales Utilizados en los Análisis Cefalométrico de Jarabak y Ricketts, Durante el Crecimiento Activo
}

\author{
Displacement of Cranial Reference Landmarks Used in Jarabak \\ and Ricketts Cephalometric Analysis During Active Growth
}

Patricia V. Díaz; Pamela Araya-Díaz* \& Hernán M. Palomino*

DÍAZ, P. V.; ARAYA-DÍAZ, P. \& PALOMINO, H. M. Desplazamiento de los puntos de referencia craneales utilizados en los análisis cefalométrico de Jarabak y Ricketts, durante el crecimiento activo. Int. J. Morphol., 33(1):229-236, 2015.

RESUMEN: El objetivo de este trabajo fue evaluar el desplazamiento de los puntos craneales: Nasion, Silla, Basion, Porion, Orbitario y Pterigoideo, utilizados como referencia en los análisis cefalométricos de Jarabak y Ricketts durante el crecimiento activo. Se seleccionaron 120 telerradiografías de perfil en formato digital, correspondientes a 60 pacientes con 2 telerradiografías cada uno, tomadas con un intervalo de tiempo mínimo de 1 año (T1 y T2), en donde T1 se encuentra antes o durante el peak de crecimiento según el Estado de Maduración Cervical Vertebral (CVM) I, II ó III de Baccetti y T2 en estadio CVM IV,V,VI (después del peak de crecimiento). Un examinador previamente calibrado, ubicó los puntos analizados y para evaluar su desplazamiento, se realizaron mediciones en T1 y T2 (3 variables para cada punto), usando como referencia 2 planos que no se modifican a partir de los 5 años de edad (LCB y Vert-T). Para determinar el desplazamiento de los puntos, se calculó la variación promedio observada entre T1 y T2 y se realizó la prueba t para muestras pareadas o Wilcoxon (según distribución) para determinar la existencia de diferencias significativas. Además, se comparó la muestra por sexo, CVM inicial y CVM final. Se encontraron variaciones entre T1 y T2 en todas las medidas, aunque sólo en 5 de ellas se encontraron diferencias significativas; no se encontró diferencias al comparar por sexo, CVM inicial y final. Es así como podemos concluir que todos los puntos craneales analizados sufren desplazamiento durante el crecimiento. Los puntos Basion y Orbitario son los que sufren mayor desplazamiento. Es necesario analizar las implicancias de estas variaciones en los resultados obtenidos de los análisis cefalométrico y evaluar la necesidad de utilizar puntos de referencia alternativos.

PALABRAS CLAVE: Puntos de referencia; Crecimiento Craneofacial; Cefalometría.

\section{INTRODUCCIÓN}

Con la introducción de la cefalometría radiológica en 1931 por Broadbent, se dió inicio a un gran desarrollo en el área de la ortodoncia, debido a que fue posible medir directamente las dimensiones esqueléticas óseas, obteniéndose una interpretación más objetiva de la morfología cráneofacial. Esto permitió el estudio de los múltiples cambios involucrados en el proceso de crecimiento y desarrollo, tanto como la evaluación de las variaciones producidas por el tratamiento de ortodoncia u ortopedia y su valoración clínica (Viazis, 1995; Celik et al., 2009).

Diversos análisis cefalométricos descritos en la literatura como Ricketts, Steiner, Jarabak, entre otros, se basan en la identificación de ciertos puntos de referencia anatómi$\cos$ o construidos en base a diversas estructuras craneofaciales, a partir de los cuales se establecen medicio- nes angulares y lineales (Aguila, 1991.) En general, estos análisis utilizan planos de referencia conformados por puntos ubicados en el cráneo como Silla-Nasion (base craneal anterior), Porión-Orbitario (Plano Frankfort), Basion-Nasion entre otros, que son considerados estables en el adulto, pero que podrían modificarse durante el período de crecimiento activo del paciente, debido a la gran cantidad de cambios que se producen en esta etapa, lo que llevaría a una variación de su posición espacial y a un posible error en la interpretación de los resultados (Ramírez \& Fernández, 2012).

Puntos Cefalométricos Craneales utilizados en los análisis cefalométricos de Jarabak y Ricketts.

Nasion: punto más anterior de la sutura fronto-nasal, ubicado sobre el plano medio sagital. Por su ubicación este punto 
de referencia se modifica debido a la remodelación provocada por el proceso de crecimiento, la aposición de hueso frontal, nasal y el desarrollo de la glabela (Tollaro et al., 1996). Además, se desplazaría vertical y sagitalmente debido al desarrollo y expansión del seno frontal. El cambio en la posición de este punto, especialmente al producirse en sentido vertical, puede originar una incorrecta interpretación del patrón de crecimiento (Quirós Alvarez, 1995; Cárdenas Jaramillo, 2003).

Basion: punto más posterior en el plano sagital, ubicado sobre el borde anterior del agujero occipital, en la porción posterior de la base de cráneo, zona en la cual se encuentra la sincondrosis esfeno-occipital que completa su cierre alrededor de los 18 años, por lo que este punto recién sería estable a partir de esa edad. Además, se ve afectado por la aposición ósea en el borde anterior del agujero occipital, lo cual puede producir variaciones (Zamora \& Duarte, 2003; Ricard \& Martínez Loza, 2005).

Porion y Orbitario: el punto de referencia llamado Porion se define como el punto más anterosuperior ubicado sobre el conducto auditivo externo; por otro lado, el punto Orbitario se define como el punto más inferior ubicado sobre el margen inferior de la órbita; ambos son utilizados para construir la línea de referencia horizontal denominada Plano de Frankfort (FH), que se usa como base para diferentes análisis, aunque no existen estudios que avalen su estabilidad. Björk \& Skieller en 1976 a través de su estudio con implantes, demostraron que el punto orbitario migra hacia craneal durante el crecimiento por aposición en el margen inferior de la órbita. Por otro lado, la modificación del punto Porion ha sido objeto de muy poca investigación (Zamora \& Duarte; Ramírez \& Fernández).

Silla: Punto de referencia correspondiente al centro de la silla turca, estructura anatómica que presenta un proceso de remodelación de su contorno, provocando un aumento de su tamaño durante la infancia, debido a la reabsorción de la zona del dorso de la silla, mientras que su pared anterior permanece estable. A medida que la pared posterior se somete a cambios de reabsorción durante el crecimiento, el punto silla se mueve gradualmente en dirección dorsal-caudal (Kjær, 2012).

La diferencia en la estabilidad de la pared anterior y posterior de la silla pueden deberse a que provienen de diferentes orígenes evolutivos.

Pterigoideo: punto más posterosuperior de la fisura pterigomaxilar. Respecto de su estabilidad, no se encontraron estudios que la evalúen (Zamora \& Duarte).

\section{Referencias craneales consideradas estables.}

Punto T: Se encuentra bien documentado en la literatura que el Punto T, definido como el punto más superior de la pared anterior de la silla turca en la unión con el tubérculo pituitario y la lámina cribosa del etmoides, permanece estable en el tiempo a partir de los 5 años de edad (Tollaro et $a l$.). Esto se explica debido a que se encuentra en la base craneal media, la cual completa su desarrollo alrededor de los 5 años de edad, como mecanismo de protección del cerebro y otros órganos vitales. Sólo el desarrollo del cerebro influye en su crecimiento, siendo absolutamente libre de la influencia de otras fuerzas de desarrollo (Viazis). Esto ha sido además, corroborado en un estudio longitudinal, realizado en el año 2010 que compara el crecimiento craneal y la maduración esqueletal, en el cual se obtuvo como resultado que el punto T, presenta un $100 \%$ de estabilidad durante todos los periodos de crecimiento, tanto en la pubertad como en la adultez (Arat et al., 2010).

Línea de base de cráneo estable (LCB): fue descrita por Tollaro et al. en 1995 y está determinada por el punto T y una tangente a la lámina cribosa del etmoides. Su estabilidad, ha sido ratificada por De Coster (2007) y Ford (1958) en sus estudios de crecimiento de la base de cráneo, donde determinó que la lámina cribosa deja de crecer en longitud anteroposterior a los dos años de edad (Ghafari et al., 1987).

Vert T: línea construida perpendicular a LCB que pasa a través del punto $\mathrm{T}$.

Dado lo anterior, y debido a la importancia de contar con puntos de referencia estables que permitan analizar la morfología cráneofacial, tanto con fines diagnósticos como para determinar el efecto de ciertas terapias, es que el objetivo del presente trabajo es evaluar la estabilidad de los puntos de referencia craneales utilizados en los Análisis Cefalométricos de Jarabak y Ricketts, y verificar si estos sufren un desplazamiento significativo durante el peak puberal.

\section{MATERIAL Y MÉTODO}

Se realizó un estudio, a partir del análisis de telerradiografías laterales de cráneo, correspondientes a individuos procedentes de una clínica de práctica privada de Ortodoncia y de la clínica de postgrado de Ortodoncia y Ortopedia Dento Máxilo Facial de la Universidad Andrés Bello, Santiago de Chile.

Se seleccionaron pacientes que contaban con dos telerradiografías de perfil tomadas con un intervalo míni- 
mo de tiempo de 1 año (T1 y T2), en donde $\mathrm{T} 1$ se encontraba en una etapa de desarrollo previa o durante al peak de crecimiento puberal y $\mathrm{T} 2$ en una etapa de desarrollo posterior al peak puberal.

Para la determinación de la etapa de desarrollo de cada individuo, se utilizó el Estado De Maduración Vertebral Cervical de Baccetti (CVM), en el cual mediante la observación de la morfología de la segunda (C2), tercera (C3) y cuarta (C4) vértebra cervical, se clasifica a los pacientes en CVM I, II, III (previo o durante el peak puberal) IV, V ó VI (post peak puberal) (Baccetti et al., 2005).

Se descartaron aquellos individuos portadores de alguna malformación cráneofacial o síndrome, y aquellos que durante el tiempo de la toma radiográfica, fueron sometidos a tratamientos de tipo ortopédico como: activadores funcionales, fuerzas extraorales y máscaras de tracción frontal, de manera de aislar la influencia del factor "tratamiento" del crecimiento propiamente tal.

En las 120 telerradiografías laterales de cráneo obtenidas en formato digital (correspondientes a 60 pacientes), se marcaron los puntos a estudiar: Silla (S), Nasion (Na), Basion (Ba), Porion (Po), Orbitario(Or) y Pterigoídeo (Pt). (Fig. 1). Se trazaron los planos de referencias construidos a partir de estructuras consideradas estables (LCB) y VertT y se obtuvieron 18 medidas lineales para evaluar el desplazamiento de los puntos (3 medidas para evaluar cada punto):

Medidas Lineales: i) T-X, ii) $\mathrm{X}-\mathrm{Y}$ y iii) Vert T-X; en las cuales $\mathrm{X}$ corresponde al punto de referencia craneométrico evaluado e $\mathrm{Y}$ a un punto ubicado en la intersección de la línea LBC y perpendicular a LBC que pasa por el punto $\mathrm{X}$.

Dado la orientación de las medidas, se consideró que Vert T-X determinaría el desplazamiento sagital, $\mathrm{X}-\mathrm{Y}$ el desplazamiento vertical y $\mathrm{T}-\mathrm{X}$ puede variar por ambos tipos de desplazamiento.

Para determinar la ubicación de puntos y planos de referencia se utilizó el programa Paint versión 6.2 de Windows 8 y para efectuar las mediciones se utilizó un programa de medición digital llamado Meazure 2.0.

Confiabilidad de los datos. Para maximizar la confiabilidad de los datos y minimizar el error, se escalaron las radiografías en base a la regla milimetrada que viene incorporada en el Cefalostato, de manera de eliminar el factor de magnificación de las mismas, y se realizó una calibración inter e intra-examinador.

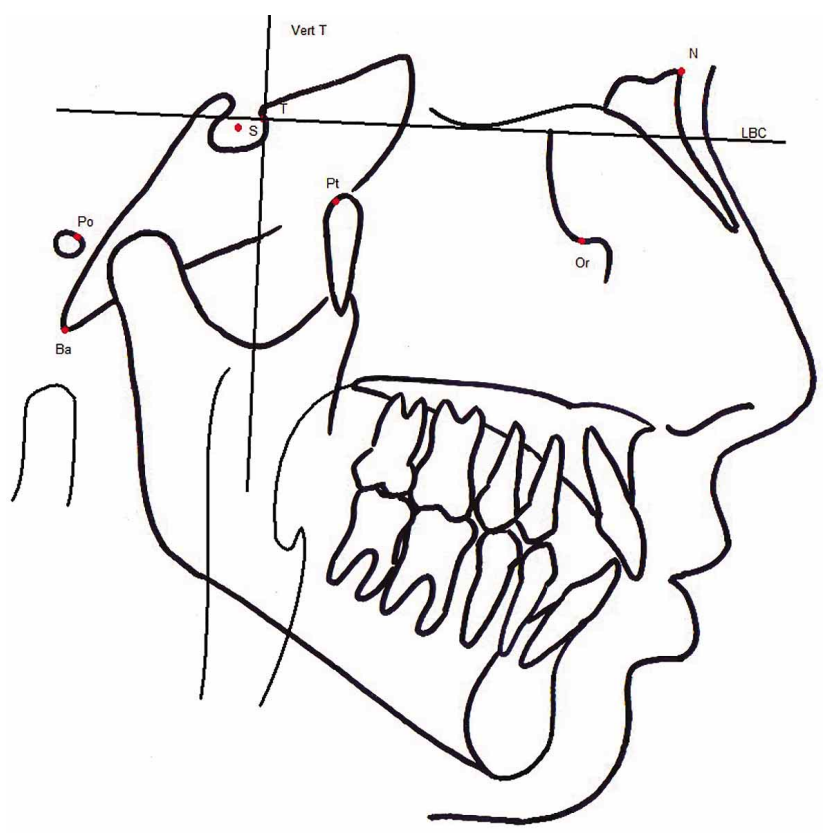

Fig. 1. Puntos de referencias Analizados: Silla (S) Nasion (N), Basion (Ba), Porion (Po), Orbitario (Or) y Pterigoideo (Pt). Punto y planos de referencia estable: Punto T, Linea LCB y Vert T.

Para la calibración inter-examinador, se seleccionaron 10 telerradiografías laterales de cráneo al azar, las cuales fueron medidas por un experto (gold standard) y el examinador. El nivel de concordancia adquirido, entre ambos, se comprobó a través del cálculo del coeficiente de correlación intra-clase (ICC).

Una vez comprobada la calibración inter-examinador, se realizó la calibración intra-examindor, para lo cual se realizó una repetición de medidas, en 10 radiografías, en 2 días no consecutivos. El nivel de concordancia adquirido, entre ambas mediciones, se comprobó también a través del cálculo del ICC.

Adicionalmente, aún cuando se realizó una calibración exitosa, las mediciones fueron realizadas tanto por el examinador como por el experto en los 30 primeros pares de telerradiografías laterales; los resultados obtenidos fueron sometidos a la prueba de Bland-Altman para determinar el grado de variación (error) entre ambas mediciones.

Análisis Estadístico. Una vez obtenidos los datos, éstos fueron tabulados en formato Excel y se realizaron los siguientes análisis utilizando el programa Prism 5 para Mac OSX, versión 5.0a:

1. Determinación de la existencia de variación en los puntos previo-durante y post peak puberal en la muestra completa: 
Una vez obtenidos los datos en T1 y T2, se realizó la prueba de Kolmogorov-Smirnoff para determinar la distribución de cada variable y se realizó la comparación de los resultados en el total de la muestra, mediante la prueba t para datos pareados o prueba de Wilcoxon según distribución.

Adicionalmente se realizó la resta aritmética de los resultados (T2-T1) y se utilizó el valor absoluto de los mismos para determinar el promedio de las diferencias y su desviación estándar.

2. Comparación según sexo: El total de la muestra se dividió en dos grupos según su sexo (masculino y femenino), y se compararon las variables por sexo, tanto en $\mathrm{T} 1$ como en $\mathrm{T} 2$, mediante una la prueba t para datos independientes o prueba de Mann-Whitney según distribución.

En las variables que resultaron tener diferencia estadísticamente significativa entre sexos, se realizó la comparación entre $\mathrm{T} 1$ y T2 en hombres y mujeres por separado.

3. Comparación según CVM inicial: El total de la muestra se dividió en tres grupos según el estado de maduración cervical observado en T1 (I, II ó III), y se compararon los datos, según CVM inicial, tanto en T1 como en T2 mediante un la prueba de ANOVA o Kruskall-Wallis según distribución.

En las variables que resultaron tener diferencia estadísticamente significativa entre los distintos CVM iniciales, se realizó la comparación entre T1 y T2 en los pa- cientes con CVM-I inicial, CVM-II inicial y CVM-III inicial por separado.

4. Comparación según CVM final: El total de la muestra se dividió en tres grupos según el estado de maduración cervical observado en T2 (IV, V ó VI), y se compararon los datos, según CVM final, tanto en T1 como en T2 mediante la prueba ANOVA o Kruskall-Wallis según distribución.

En las variables que resultaron tener diferencia estadísticamente significativa entre los distintos CVM finales, se realizó la comparación entre T1 y T2 en los pacientes con CVM-IV final, CVM-V final y CVM-VI final por separado.

\section{RESULTADOS}

La muestra estuvo conformada por 60 pares de telerradiografías laterales, correspondientes a 60 pacientes, 31 mujeres y 29 hombres, con un promedio de edad en T1 de 11 años 1 mes ( \pm 18 meses) y en T2 14 años 9 meses ( \pm 18 meses). El 43,3\% de los pacientes en $\mathrm{T} 1$ se encontraban en CVM III, $35 \%$ en CVM II y $21,7 \%$ en CVM I, mientras que en T2 58,3\% se encontraba en CVM V, 33,3\% en CVM IV y $8,4 \%$ en CVM VI. El intervalo de tiempo transcurrido entre la primera (T1) y segunda (T2) teleradiografía varió entre 1 año a 8 años 5 meses, con un promedio de 4 años \pm 23 meses.

Confiabilidad de Datos. En la calibración, el ICC obtenido tanto en la calibración inter-examinador como intra-exami-

Tabla I. Diferencia aritmética promedio entre T1 y T2 y error según la prueba de Bland Altman.

\begin{tabular}{llcccc}
\hline \multirow{2}{*}{ Punto } & Variables & $\begin{array}{c}\text { Promedio de } \\
\text { la diferencia }\end{array}$ & DE & Bland-Altman & p \\
\cline { 1 - 1 } N & T-N & 2,56 & 1,90 & 0,04 & $0,005^{*}$ \\
& VertT-N & 2,61 & 1,93 & 0,02 & $0,011^{*}$ \\
\multirow{3}{*}{ S } & N-Y & 2,20 & 1,74 & 0,02 & 0,654 \\
& T-S & 0,58 & 0,48 & 0,01 & 0,215 \\
& S-Y & 0,68 & 0,68 & 0,02 & 0,228 \\
\multirow{3}{*}{ B } & VertT-S & 1,14 & 4,57 & 0,01 & 0,506 \\
& T-Ba & 3,00 & 3,31 & 0,04 & $0,048^{*}$ \\
& Ba-Y & 3,14 & 3,08 & 0,05 & 0,976 \\
Po & VertT-Ba & 3,01 & 3,15 & 0,02 & 0,439 \\
& T-Po & 2,41 & 2,46 & 0,03 & 0,536 \\
& Po-Y & 2,62 & 2,87 & 0,006 & 0,409 \\
Or & VertT-Po & 2,48 & 2,05 & 0,01 & $<0,0001^{*}$ \\
& T-Or & 3,24 & 3,63 & 0,01 & $0,031^{*}$ \\
& Or-Y & 2,69 & 2,97 & 0,005 & 0,119 \\
\multirow{2}{*}{ Pt } & VertT-Or & 3,28 & 3,41 & 0,01 & 0,111 \\
& T-Pt & 1,44 & 1,24 & 0,02 & 0,682 \\
& Pt-Y & 1,62 & 1,35 & 0,015 & $<0,0001^{*}$ \\
& VertT-Pt & 1,36 & 1,50 & 0,01 & 0,577 \\
\hline
\end{tabular}


nador fue mayor a 0,9 para todas las variables, lo que es considerado como un acuerdo casi perfecto. En el cálculo de grado de error, los resultados del análisis de Bland-Altman para cada variable se muestran en la Tabla I. Este fue menor a 0,06 para todas las variables.

Resultados previo-durante y post peak puberal. Comparación T1-T2 muestra completa: Al realizar la comparación de las variables lineales entre T1-T2 en la muestra completa, se encontraron diferencias estadísticamente significativas en 5 variables (Tabla I): T-N, T-Ba, Pt-Y, T-Or, VertT$\mathrm{N}$ y VertT-Po.

Diferencia Aritmética T1-T2 en la muestra completa (Tabla I): Al obtener el promedio de los valores absolutos de la diferencia T1-T2, se obtuvo que, 6 de las variables presentan una diferencia promedio entre 0,2 y $12 \mathrm{~mm}$, con una diferencia promedio entre 2 y $4 \mathrm{~mm}$.

\section{DISCUSIÓN}

Un correcto diagnóstico, es esencial para la planificación de un adecuado tratamiento, lo que lleva a su vez a un mejor pronóstico para el paciente frente a su patología o maloclusión. Para esto, es fundamental efectuar un análisis cefalométrico que nos proporcione la información necesaria, con un alto porcentaje de validez y confiabilidad (Arat et al., 2010; Baccetti et al.).

Existen variadas fuentes que pueden llevar a algún grado de error al realizar los análisis cefalométricos; algunos de ellos son inherentes a la técnica y en parte es inevitable que ocurran, sin embargo, se puede tener ciertas consideraciones para que se expresen mínimamente, como utilizar puntos de referencia estables y una adecuada y estandarizada calibración (Arat et al., 2010) lo que fue considerado en este trabajo, comprobándose que las mediciones realizadas son confiables y presentan un mínimo error $(<0,06)$.

El uso de estructuras estables es fundamental para evaluar cambios y estudiar el desarrollo craneofacial de forma longitudinal (Goel et al., 2004). Es por esta razón, que en numerosos estudios se utiliza el punto $\mathrm{T}$ y la lámina cribosa del etmoides como puntos y líneas de referencias estables, y por ello también, fueron utilizadas en el presente estudio.

Es importante mencionar, que el crecimiento cráneofacial se estudia generalmente en relación a la edad cronológica (Arat et al., 2001), sin embargo, ésta no siempre permite valorar el desarrollo y la maduración somática del paciente, ya que la relación entre la maduración ósea y la edad son variables e inherentes a cada individuo, por otro lado, tanto la velocidad como el ritmo de crecimiento no siguen un patrón establecido en relación a la edad. Baccetti et al., han demostrado que el estado de maduración ósea, determinado a través de la morfología de las vértebras cervicales (CVM) se puede utilizar como un indicador biológico confiable (Thilander et al., 2005; D’Escriván de Saturno, 2007).

Es por este motivo que las telerradiografías utilizadas en este estudio fueron seleccionadas y agrupadas en base al estado de maduración vertebral cervical según Baccetti et al.

En cuanto al desplazamiento de los puntos durante el crecimiento activo, se determinó la existencia de desplazamiento en todos los puntos estudiados, tal como se señala a a continuación:

Punto N: Existe un desplazamiento sagital significativo de 2,6 $\pm 1,9 \mathrm{~mm}$ promedio, durante el crecimiento activo, en cuanto al desplazamiento vertical se observa una diferencia de 2,2 $\pm 1,74 \mathrm{~mm}$, la cual sin embargo, no resultó ser estadísticamente significativa. Esto no coincide con lo planteado por otros autores, que señalan que el punto $\mathrm{N}$ se modifica significativamente tanto en sentido vertical como sagital (Arat et al., 2003). En cuanto a la magnitud del desplazamiento, este fue mayor a lo observado por Arat et al. (2003), en un estudio que evalúa el desplazamiento de puntos de referencias a través de distintos métodos de superposición, en el cual se observó una variación de entre 1,21 y 1,99 mm promedio, lo cual podría deberse a que en el estudio antes citado, el intervalo de tiempo entre T1 y T2 fue de 2 años promedio, mientras que en el presente estudio el tiempo promedio entre radiografías fue de 4 años. Este desplazamiento del punto Nasion sería producido por el crecimiento de la sutura frontonasal, a la aposición de hueso frontal, nasal y el desarrollo de la glabela (Kuroe et al., 2004; Chutimanutskul et al., 2007; Tollaro et al.).

Punto S: en relación a este punto, no se encontró diferencia significativa en ninguna de las variables medidas, teniendo un promedio de variación sagital de $1,14 \pm 4,5 \mathrm{~mm}$ y vertical menor a $0,7 \mathrm{~mm}$, a diferencia del estudio de Arat et al. (2003), en que se encontró un desplazamiento significativo tanto en sentido sagital como vertical, aunque de menor magnitud (sagital: 0,45-0,52 mm; vertical 0,94 mm), lo cual se debería al menor tiempo de observación. Esta modificación estaría relacionada con la reabsorción que sufre el dorso de la silla durante el periodo de crecimiento, en que el punto S se desplaza gradualmente hacia posterior con el aumento del tamaño de la glándula pituitaria (Kuroe et al.; Kjær).

Punto Ba: Se observó una variación tanto sagital como vertical de $3 \pm 3 \mathrm{~mm}$, sin embargo sólo resultó ser significativa 
la variación en la distancia $\mathrm{T}-\mathrm{Ba}(3 \mathrm{~mm})$, la cual varia tanto por desplazamientos en sentido sagital como vertical. Esto supera lo encontrado por Arat et al. (2003), donde el punto Basion sufre un desplazamiento significativo de entre 1,22 a $1,43 \mathrm{~mm}$ vertical y de entre 0,66 a 1,66 mm según el método de superposición utilizado, lo que puede deberse a las distintas metodologías utilizadas y al menor tiempo de observación entre radiografías. Este desplazamiento se debería a que esta porción de la base de cráneo aumenta su longitud por la actividad de la sincondrosis esfeno-occipital, la cual termina su cierre alrededor de los 18 años de edad (Zamora \& Duarte; Ricard \& Martínez Loza). Además, su variación espacial también se ve contribuida por la aposición ósea en el borde anterior del agujero occipital (Cárdenas Jaramillo).

Ricketts, utiliza este punto en su análisis y lo sugiere debido a que su uso en lugar del punto $\mathrm{S}$ ayudaría a tener en cuenta el crecimiento divergente de las estructuras (Greiner et al., 2004), sin embargo Arat et al. (2010) en un estudio longitudinal, determinó que el punto Basion era el punto más variable de la base de cráneo, con un $0 \%$ de estabilidad.

Punto Or: Se observó una variación tanto sagital $(3,28 \pm 3,41$ $\mathrm{mm})$ como vertical $(2,69 \pm 2,97 \mathrm{~mm})$, sin embargo sólo resultó ser significativa la variación en la distancia T-Or $(3,24 \pm 3,63 \mathrm{~mm})$, la cual varia tanto por desplazamientos en sentido sagital como vertical, esto concuerda con lo encontado por Bjork \& Skieller en 1976, quienes demostraron a través de implantes, que el punto Orbitario migra durante el crecimiento como resultado de la aposición en el margen orbital inferior. En cuanto a la magnitud del desplazamiento, este fue mayor a lo observado por Arat et al. (2003), en el cual se observó una variación sagital de entre 0,44 a $1,42 \mathrm{~mm}$ y vertical de entre 0,76 y $1,39 \mathrm{~mm}$ promedio producto del menor tiempo de observación de dicho estudio. En otro estudio realizado por Greiner et al., en el que se utilizaron dos grupos de cráneos, se observó un desplazamiento vertical similar $(3,9 \mathrm{~mm})$, pero un despazamiento sagital mucho mayor $(11,8 \mathrm{~mm})$, lo cual podría deberse a que se realizó la comparación entre un grupo de 2,5 a 5 años y uno de entre 18 a 20 años, un intervalo mucho mayor al del presente estudio. Además, para estimar este desplazamiento, se utilizó la distancia Or-PoC (centro del porion) punto que también podría modificarse con el crecimiento.

Punto Pt: experimenta un desplazamiento vertical significativo promedio de $1,62 \pm 1,35 \mathrm{~mm}$, en cuanto al desplazamiento sagital se observa una diferencia de $1,44 \pm 1,24 \mathrm{~mm}$, la cual sin embargo, no resultó ser significativo, a diferencia del estudio de Arat et al. (2003), en que se encontró un desplazamiento significativo tanto en sentido sagital como vertical, aunque de menor magnitud (sagital: 0,66 mm; vertical 0,48$0,53 \mathrm{~mm}$ ), lo que puede deberse a las distintas metodologías utilizadas y al menor tiempo de observación.

Punto Po: presenta un desplazamiento significativo en sentido sagital de 2,48 $\pm 2,05 \mathrm{~mm}$ en cuanto al desplazamiento vertical se observa una diferencia de $2,62 \pm 2,87 \mathrm{~mm}$, la cual sin embargo, no resultó ser significativa. En el estudio de Arat $e t$ al. (2003) no se evalúa la estabilidad de este punto y en el estudio de Greiner et al., se evaluó el punto porion centro $(\mathrm{PoC})$, no el punto Po propiamente tal, por lo que no se pueden establecer comparaciones respecto de la variación observada en este punto.

Cabe destacar que si bien la mayoría de las variables no presentaron una diferencia significativa, presentan una variación milimétrica que puede tener significación clínica, puesto que si un punto utilizado en un análisis cefalométrico presenta un desplazamiento de $3 \mathrm{~mm}$, podría provocar un cambio en el valor de las medidas cefalométricas, lo que alteraría la interpretación del análisis y afectaría el diagnóstico del paciente (Ustrell Torrent \& Durán von Arx, 2002). La variación de los puntos puede llevar también a variaciones en las mediciones angulares utilizadas en los analisis cefalométricos, en que una diferencia entre $\operatorname{los} 3^{\circ}$ y $5^{\circ}$, podría alterar la interpretación de la cefalometría, puesto que el valor obtenido puede ser producto del desplazamiento del punto de referencia provocado por los procesos de crecimiento y desarrollo, proporcionándonos un diagnóstico errado, tal como declarar una alteración donde no la hay, o por el contrario no detectar alguna patología (Ustrell Torrent \& Durán von Arx).

En cuanto a la comparación por sexo, no se obtuvieron diferencias estadísticamente significativas, lo cual no concuerda con un estudio que se realizó para determinar el tipo de crecimiento en la población de Yucatan, en que se obtuvo que el ángulo Silla en relación al polígono de Bjork en los niños, aumenta entre los 7 y 11 años de edad donde se estabiliza, en cambio en las niñas a esta edad oscila entre los $127-126^{\circ}$, aumentando bruscamente a los 11 años donde alcanza un valor de $130^{\circ}$, llegando a los 14 años a obtener el mismo valor que a los 7 años (Hérnandez Tello \& Tello López, 1998). No se encontraron estudios que presenten información sobre el desplazamiento de los puntos según sexo.

Al comparar según el estado de maduración cervical inicial, se obtuvo diferencia significativa solo para la variables T-Ba, lo cual pudiera influir en el resultado, ya que en teoría, al encontrarse el niño en etapas más tempranas de crecimiento, el desplazamiento de los puntos podría ser mayor, sin embargo, no se encontraron diferencias estadísticamente significativas al compararar T1 vs. T2 separadamente en CVM I, CVM II y CVM III inicial en dicha variable. 
Por último, al realizar la comparación según el Estado de maduración Cervical final (en T2) se obtuvo diferencia significativa para la variable $\mathrm{T}-\mathrm{Ba}$ y VertT-Ba, no observándose diferencias significativas en ninguna de ellas al comparar T1 vs. T2 por separado.

\section{CONCLUSIONES}

Todos los puntos craneales utilizados como referencia en los análisis cefalométricos de Jarabak y Ricketts su- fren desplazamiento durante el peak de crecimiento. Los punto Basion y Orbitario son los puntos de referencia que sufren mayor desplazamiento. Las diferencias respecto de otros estudios podrían atribuirse a las distintas metodologías utilizadas y a la variación en el intervalo de tiempo entre las observaciones.

Se hace necesario analizar las implicancias de estas variaciones en los resultados obtenidos del análisis cefalométrico y evaluar la necesidad de utilizar puntos de referencia alternativos.

DÍAZ, P. V.; ARAYA-DÍAZ, P. \& PALOMINO, H. M. Displacement of cranial reference landmarks used in Jarabak and Ricketts cephalometric analysis during active growth. Int. J. Morphol., 33(1):229-236, 2015.

SUMMARY: The objective of this study was to evaluate the displacement of cranial reference points: Nasion, Sella, Basion, Porion, Orbitale and Pterygomaxillary, used in Jarabak and Ricketts cephalometric analysis, during active growth. Hundred and twenty digitalized lateral telerradiographies, corresponding to 60 patients ( 2 teleradiographies each one), were collected. The radiographies were taken with a minimum interval of one year between them (T1 and T2), where T1 is taken before or during the pubertal growth peack according to the cervical vertebral maturation stages developed by baccetti (CVM) I, II or III and T2 in CVM IV,V,VI (after the growth peak). Then, a previously calibrated examinator marked reference points and cephalometric measurements were taken (2 variables for each landmark). Measurements were made using craniofacial stable structures as references (stable basicranial line and Vertical T). To detect displacement in the landmark positions, $\mathrm{t}$ test or Wilcoxon test according to the distribution of each variable, was used to compare the data between T1 and T2. Also, comparisons were made by sex, and by initial and final CVM. All of the variables have variations between $\mathrm{T} 1$ y T2, but only 5 have a statistically significant difference. There were no differences between sexes and at initial and final CVM. In conclusion, all of the reference landmarks analyzed had displacement during active growth. Point Basion and Orbitale suffered the largest displacement. It is necessary to analyze the clinical implications of this displacement in order to evaluate the convenience of using alternative reference landmarks.

KEY WORDS: Reference points; Landmarks; Craniofacial growth.

\section{REFERENCIAS BIBLIOGRÁFICAS}

Aguila, J. F. Crecimiento Craneofacial: Ortodoncia y Ortopedia. Sabadell, Aguiram, 1991.

Arat, M.; Köklü, A.; Ozdiler, E.; Rübendüz, M. \& Erdogan, B. Craniofacial growth and skeletal maturation: a mixed longitudinal study. Eur. J. Orthod., 23(4):355-61, 2001.

Arat, Z. M.; Rübendüz, M. \& Akgül, A. A. The displacement of craniofacial reference landmarks during puberty: a comparison of three superimposition methods. Angle Orthod., 73(4):37480, 2003.

Arat, Z. M.; Türkkahraman, H.; English, J. D.; Gallerano, R. L. \& Boley, J. C. Longitudinal growth changes of the cranial base from puberty to adulthood. A comparison of different superimposition methods. Angle Orthod., 80(4):537-44, 2010.

Baccetti, T.; Franchi, L. \& McNamara, J. A. Jr. The cervical vertebral maturation (CVM) method for the assessment of optimal treatment timing in dentofacial orthopedics. Semin. Orthod., 11(3):119-29, 2005.
Cárdenas Jaramillo, D. Fundamentos de Odontología: Odontología Pediátrica. $3^{\mathrm{a}}$ ed. Medellin, Corporación para investigación Biológica, 2003. pp.265-71.

Celik, E.; Polat-Ozsoy, O. \& Toygar Memikoglu, T. U. Comparison of cephalometric measurements with digital versus conventional cephalometric analysis. Eur. J. Orthod., 31(3):241-6, 2009.

Chutimanutskul, W.; Geenty, J. P.; Shen, G. \& Darendeliler, M. A. Validity and reliability of ear landmarks as reference points for cephalometric analysis. World J. Orthod., 8(2):122-8, 2007.

De Coster, L. The familial line, studied by a new line of reference. Eur. J. Orthod., 29(Suppl. 1):i65-7, 2007.

D’Escriván de Saturno, L. Ortodoncia en Dentición Mixta. Caracas, Amolca, 2007. pp.106-15.

Ford, E. H. R. Growth of the human cranial base. Am. J. Orthod., 44(7):498-506, 1958. 
Greiner, P.; Müller, B. \& Dibbets, J. The angle between the Frankfort horizontal and the sella-nasion line. Changes in porion and orbitale position during growth. J. Orofac. Orthop., 65(3):217$22,2004$.

Ghafari, J.; Engel, F. E. \& Laster, L. L. Cephalometric superimposition on the cranial base: a review and a comparison of four methods. Am. J. Orthod. Dentofacial Orthop., 91(5):403-13, 1987.

Goel, S.; Bansal, M. \& Kalra, A. A preliminary assessment of cephalometric orthodontic superimposition. Eur. J. Orthod., 26(2):217-22, 2004.

Hérnandez Tello, F. J. \& Tello López, T. Determinación del tipo de crecimiento facial en niños yucatecos. Rev. Asoc. Dent. Mex., 55(4):191-201, 1998.

Kjær, I. Sella turcica morphology and the pituitary gland-a new contribution to craniofacial diagnostics based on histology and neuroradiology. Eur. J. Orthod., 37(1):28-36, 2015.

Kuroe, K.; Rosas, A. \& Molleson, T. Variation in the cranial base orientation and facial skeleton in dry skulls sampled from three major populations. Eur. J. Orthod., 26(2):201-7, 2004.

Quirós Alvarez, O. La base anterior del cráneo, consideraciones en inclinación y longitud. Acta Odontol. Venez., 33(1), 1995. Disponible en: http://www.actaodontologica.com/ oscar_quiros/base_anterior_craneo.asp

Ramírez, C. J. A. \& Fernández, L. N. Estudio cefalométrico de la estabilidad del plano de Frankfurt intracraneal durante el proceso de crecimiento y desarrollo craneofacial. Rev. Chil. Ortod., 29(2):71-81, 2012.

Ricard, F. \& Martínez Loza, E. Osteopatía y pediatría. Madrid, Médica Panamericana, 2005.

Thilander, B.; Persson, M. \& Adolfsson, U. Roentgencephalometric standards for a Swedish population. A longitudinal study between the ages of 5 and 31 years. Eur. J. Orthod., 27(4):370-89, 2005.

Tollaro, I.; Bacetti, T. \& Franchi, L. Mandibular skeletal changes induced by early functional treatment of Class III malocclusion: a superimposition study. Am. J. Orthod. Dentofacial Orthop., 108(5):525-32, 1995.

Ustrell Torrent, J. \& Durán von Arx, J. Ortodoncia. $2^{\text {a }}$ ed. Barcelona, Edicions Universitat Barcelona, 2002.

Viazis, A. D. Atlas de Ortodoncia. Principios y Aplicaciones Clínicas. Buenos Aires, Médica Panamericana, 1995.

Zamora, C. \& Duarte, S. Atlas de cefalometría: análisis clínico y práctico. Caracas, Amolca, 2003.

\author{
Dirección para Correspondencia: \\ Pamela Araya \\ Departamento del niño y adolescente \\ Área de Ortodoncia, Facultad de Odontología \\ Universidad Andrés Bello \\ Calle Echaurren 237 \\ Santiago \\ CHILE
}

Email: payleen@gmail.com

Recibido : 08-10-2014

Aceptado: 19-01-2015 